EUROPEAN ORGANIZATION FOR NUCIFAR RESEARCH

CERN-LEP-RT/89-27 and CLIC Note 90

CERN LIBRARIES, GENEVA

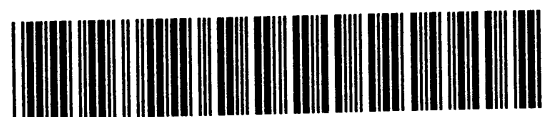

CM-P00064516

Comparison between Smooth and AG Focusing for the CLIC Main Linac

H. Henke and J. Tückmantel

Presented at the 1989 Particle Accelerator Conference 20-23 March 1989, Chicago, I.S.A.

Geneva

March 1989 


\title{
Comparison between Smooth and AG focusing for the CLIC Main Linac
}

\author{
H. Henke and J. Tückmantel \\ CERN, Geneva, Switzerland
}

\begin{abstract}
Future linear colliders with extremely low emittance beams seem to require strong BNS damping in order to cope with wake field effects. Under this condition the smooth focusing approximation is over-optimistic as compared to AG focusing. For CLIC it is found that the emittance blow-up due to wake fields in a non-perfect machine is about one order of magnitude smaller for smooth than for AG focusing.
\end{abstract}

\section{Introduction}

Very low emittance beams, in the order of $10^{-6} \mathrm{mrad}$, as required for high energy linear colliders, are easily degraded by machine imperfections. The worst errors are thereby lateral position errors of quadrupoles. They exert dipole kicks on the particles which then start betatron oscillations. If the errors are statistically independent the betatron amplitude will increase with $\sqrt{N_{q}}$ ( $N_{q}$ number of quadrupoles) and the phase is random. In the case of a highly relativistic bunch the situation is similar: the bunch can be divided longitudinally into slices and each slice behaves like a single particle in the focusing channel. The inevitable energy spread between slices and the undefined betatron phase cause an emittance dilution. For slowly varying errors, steering of the beam could be envisaged to get rid of the phase problem. But even then the oscillating head of the bunch creates wake fields which blow up the tail.

A similar effect comes from laterally displaced RF structures. The difference is that the bunch head does not experience any kick and stays on-axis. The tail gets wake fieli kicks which are statistically independent.

In linear colliders which tend to have a high RF frequency the beam blow-up due to wake fields is normally very strong. Therefore it seems unavoidable to rely on BNS\{1] damping. If the damping is properly adjusted the motion of the bunch core stays coherent. So, not only is the blow-up defeated but also chromatic effects are reduced. These effects have been calculated and tolerances on different machine errors were established[2]|3| for the CERN Linear Collider, CLIC. The calculations were done with the integration code LINBUNCH assuming smooth focusing. In this paper we will make a comparison with AG focusing results[4] obtained with the tracking code LTRACK[5].

\section{BNS Damping}

Transverse wake fields created by the head of an off-axis travelling bunch kick the core and tail even further away from the axis. The bunch break-up has an exponentially growing amplitude and an increasing betatron phase lag from head to tail. . It can be stabilized or even damped by introducing a positive spread in phase advance from head to tail[2]. The criterion|6] for complete wake field compensation can be written as

$$
2 k(z, s) \frac{\partial}{\partial z} k(z, s)=\frac{e Q}{E(s)} \frac{d}{d z} W_{T}(z)
$$

with $k(z, s)$ the wave number in the smooth focusing approximation, $E(s)$ the energy, $Q$ the total charge in the bunch and $W_{\perp}(z)$ the transverse wake potential. 8 is the coordinate along the linac and $z$ is the longitudinal position in the bunch going from head to tail. From equ. 1:
- In order to fulfill the criterion for all $s$, the lattice, i.e. the betatron wavelength $\lambda_{\rho}$ has to scale with $E(s)^{-1 / 2}$.

- The relative spread in k scales with $\lambda_{\beta}^{2}$.

In reality the criterion, equ. 1 , can only be fulfilled approximately and only over the core of the bunch. Two ways are known for creating damping. The first way, which we shall call $A$, is to introduce an energy spread within the bunch by placing it on the rising slope of the RF voltage|5|. The required energy spread is (in linear approximation)

$$
\sigma_{E}=\frac{e Q}{8 \pi^{2} \xi} W_{T}^{\prime} \sigma_{\&} \frac{\lambda_{\rho}^{2}}{E}
$$

where $W_{T}^{\prime}$ stands for the slope of the wake potential in the bunch center. This method is proved to work[7] but has a couple of disadvantages. The spread in $k$ is small for reasonable RF phase angles. Therefore very strong focusing is needed with $\lambda_{\beta_{0}}$ between 5 and $10 \mathrm{~m}$ for the CLIC parameters. The high energy spread has to be reduced by switching the RF phase from the rising to the falling slope in the second half of the machine. As a result the bunch blows up slightly and suffers a loss in voltage gain. The final energy spread is higher than the possible minimum.

$A$ second way, called $B$, to meet the damping criterion is to use a focusing system which acts on the bunch scale. This is what an RF quadrupole system does. For our purposes where we need a spread in phase advance we will combine RFQs, operated at zero crossing of the gradient, with an external quadrupole channel providing the focusing[8]. The ratio $\alpha$ of peak $R F Q$ gradient to external gradient necessary for damping is then

$$
\alpha=\frac{e Q}{16 \pi^{3} \xi} W_{T}^{\prime} \lambda_{R F} \frac{\lambda_{\beta}^{2}}{E}
$$

(again in linear approximation). The advantages of method B compared to $A$ are the following. For reasonably small $\alpha$, between 0.5 and 1 , the spread in $k$ is larger and the focusing strength can therefore be reduced. The machine becomes less sensitive to quadrupole position errors. The damping is maintained over the whole machine while the RF phase angle is chosen as the one yielding minimum energy spread.

\section{Simulation Results}

All numerical results refer to a hypothetical $1+1 \mathrm{TeV}$ collider CLIC which is under study at CERN/9]. The parameters are given in Table 1. Cases $A$ and $B$ refer to machines where BNS damping is obtained via energy spread or with RFQs. The corresponding betatron wavelengths were found by a trade-off between good damping conditions and reasonable values either for energy spread or for RFQ gradient. Tolerances are always given for $25 \%$ emittance increase. They are only approximate values, since complete statistics could not always be done for reasons of computer time consumption.

Two types of machine error are distinguished: static or slowly varying errors and fast varying errors. The first, called alignment errors, can, at least in principle, be corrected by realignment and feedback systems. The second type, called jitter errors, are uncorrectable. 
Energy range

Accelerating gradient

RF frequency

RF structure length, aperture

No. of particles per bunch

R.m.s. bunch length

Transverse wake potential

slope at bunch centre

Lattice:

magnet and cell length

Normalized emittance

Betatron wavelength,

B $\lambda_{\beta 0}=20 \mathrm{~m}$ to $\lambda_{\beta f}=282.8 \mathrm{~m}$

$\begin{array}{ll}\text { B } & \lambda_{\beta 0}=20 \mathrm{~m} \text { to } \lambda_{\beta \rho}= \\ \text { B } & N_{q}=2828, N_{q}=707\end{array}$

Table 1: CLIC parameters used in this paper

\section{Lateral Position Jitter of Quadrupoles}

Laterally displaced quadrupoles exert dipole kicks on the bunch and blow up the betatron amplitude. If there were no wake fields the blow-up would be incoherent due to the spread in phase advance within the bunch.

With wake fields and optimum damping the bunch core is kept roughly coherent, as shown in Fig. 1 a for machine $A$. The RF phase, yielding minimum emittance blow-up, was found to be $-3^{\circ}$ with a resulting $\sigma_{E}=1.8 \%$, in good agreement with the theoretical value of $1.6 \%$ calculated from equ. 2 . The energy spread can be reduced to $1.1 \%$ by switching the RF phase from $-3^{\circ}$ to $+5^{\circ}$ in the second half of the machine. Then, the tolerable r.m.s. quadrupole jitter is $0.16 \mu \mathrm{m}$ in the smooth focusing case. With AG focusing the resulting betatron amplitude is ten times larger and the emittance increase 25 times larger and the tolerable quadrupole jitter is only $0.03 \mu \mathrm{m}$. It is possible to make the machine less sensitive by reducing the focusing strength. But since $\sigma_{E} \sim \lambda_{\beta}^{2}$, one cannot increase $\lambda_{\beta O}$ much above $10 \mathrm{~m}$ where only a factor 1.5 is gained in tolerance and the loss in voltage gain is $15 \%$.

The situation improves appreciably when an RFQ system (machine $B$ ) is added on. For $\lambda_{\beta 0}=20 \mathrm{~m}$ optimum damping was found at $\alpha=0.7$. The RF phase angle was set to $+5^{\circ}$ yielding a minimum energy spread of $\sigma_{E}=0.8 \%$. As can be seen in Fig. 1b, the core of the bunch stays very coherent. Under these conditions the tolerable quadrupole jitter is $1.2 \mu \mathrm{m}$ in the smooth focusing approximation and $0.2 \mu \mathrm{m}$ for AG focusing. Again we tried to reduce the focusing strength even further but no essential reduction of tolerances was found. Apparently the reduced sensitivity due to a larger betatron amplitude was made up by a less effective damping mechanism.

The $\alpha$ value of 0.7 found in simulations is smaller than the theoretical value 2 calculated from equ. 3 . This happens under strong damping conditions where non-linearities play an important role.

\section{Lateral Position Jitter of RF Sections}

Laterally displaced RF structures are normally less critical than displaced quadrupoles. The head of the bunch stays aligned and the tail gets random wake field kicks proportional to the structure displacement. Due to the randomness of the kicks BNS damping is not effective in the usual sense. It is a stochastic process where the slowly blown up tail lags behind in betatron phase. BNS damping, therefore, works only in average and requires a different set of parameters.

Since the position tolerances for the RF sections are less stringent than for quadrupoles, we keep the same parameters as in the above section. Then for both machines $A$ and $B$ the tolerable RF section jitter is about $10 \mu \mathrm{m}$ for smooth as well as AG focusing.

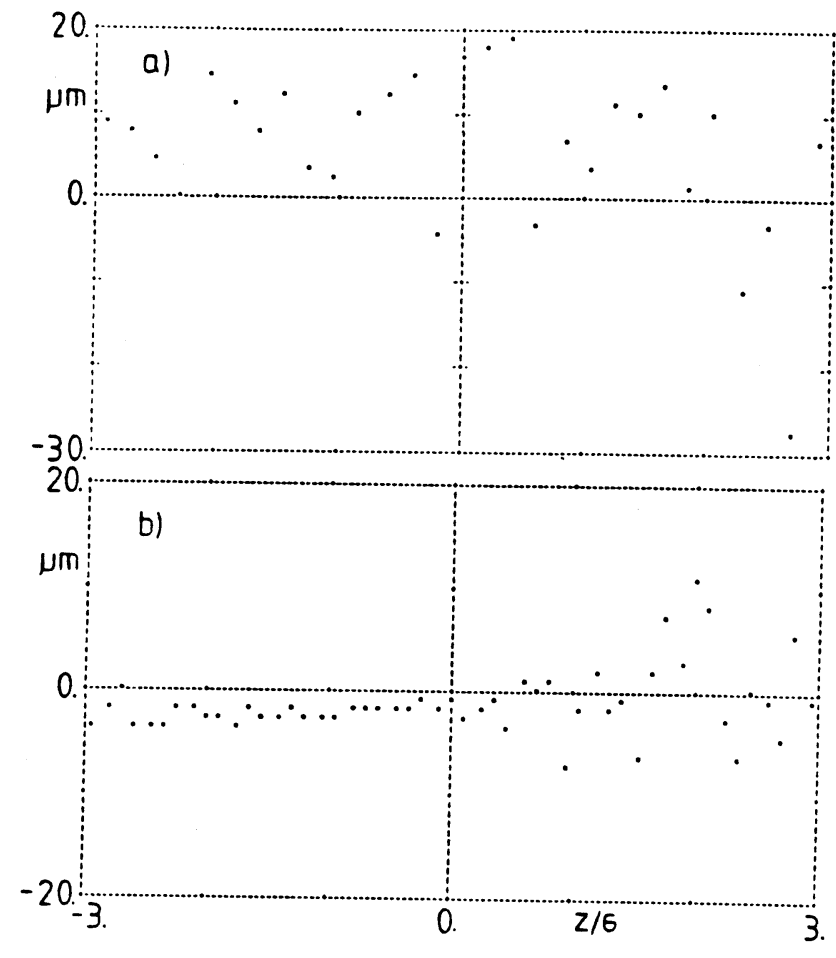

Figure 1: Position of slice centroids of a bunch at the end of the linac (smooth focusing, lateral quadrupole jitter of $1 \mu \mathrm{m}$ r.m.s. value). a) machine $A, b)$ machine $B$

\section{Lateral Quadrupole Alignment Errors}

The very tight tolerances on the quadrupole jitter clearly indicate that static or slowly varying errors have to be corrected. Without correction the dipole kicks of displaced quadrupoles blow up the motion of the centre of charge following $\sqrt{N_{e}}$ (Fig. 2a). With correction the amplitude is bound to a value corresponding to the effect of a single quadrupole displaced by the r.m.s. value (Fig. 2b). A perfect correction means detecting reliably errors of $1 \mu \mathrm{m}$ which is hard to imagine. But instead of correcting every individual element a feedback system is conceivable which corrects on average. It detects the increasing amplitude of the centre of charge motion and corrects the focusing with an evolutionary strategy in such a way that the amplitude stays bound. In a simulation calculation this is very time-consuming to do. We therefore make the optimistic assumption that every error is detected and corrected with correction coils on the two following quadrupoles. An equivalent solution to corrections coils would be replacing every quadrupole displacement $\Delta x_{i}$ by the new value $\Delta x_{i}-2 \Delta x_{i-1}+\Delta x_{i-2}$ in the case of smooth focusing. In this algorithm the second term inverts the slope of the trajectory caused by the dipole kick of quadrupole $i-1$, and the third term zeroes the trajectory caused by quadrupole $i-2$. With the same RF parameters as in above, the allowable alignment errors are $3.2 \mu \mathrm{m}$ for machine $A$ and $12.2 \mu \mathrm{m}$ for machine $B$ in the case of smooth focusing. With AG focusing we again lose a factor of about 10 yielding $0.5 \mu \mathrm{m}$ for machine $A$ and $1 \mu \mathrm{m}$ for machine $B$. Figure 3 shows the beam shape and phase plot for the last case.

\section{Injection Offset}

As a last example we look at an error at injection. This is the most convincing way of demonstrating the power of BNS damping. Figure $4 \mathrm{a}$ shows the centre of charge motion of a bunch which is injected with $10 \mu \mathrm{m}$ offset into machine A with smooth focusing. After a few hundred meters the motion has died away. For machine $B$, with RFQs, the motion is already completely 


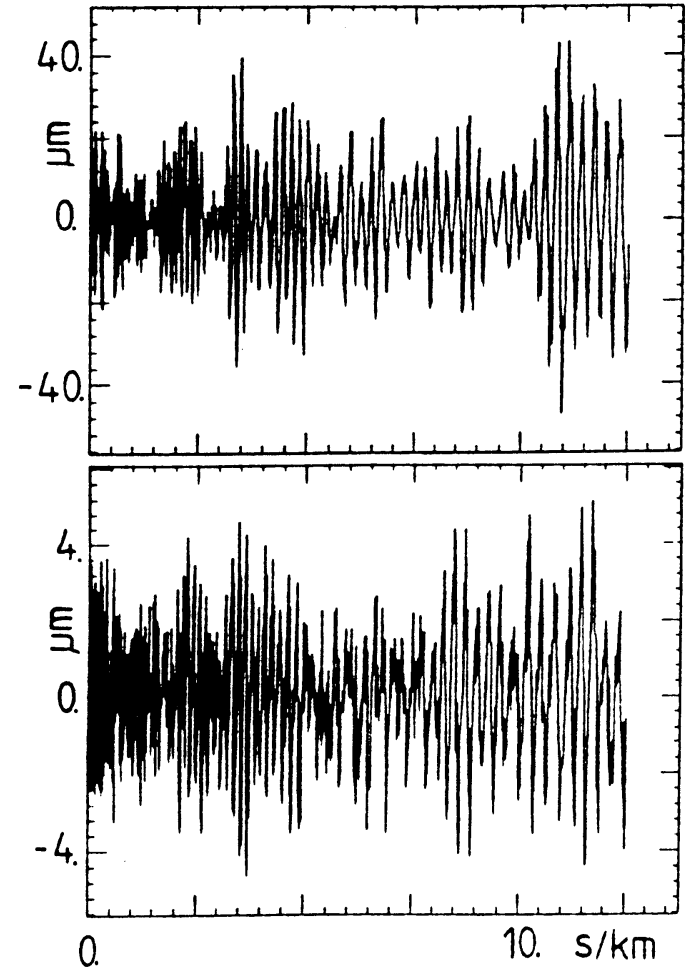

Figure 2: Centre of charge motion along the linac (AG focusing, lateral quadrupole jitter of $1 \mu \mathrm{m}$ r.m.s. value). a) random errors, b) corrected random errors

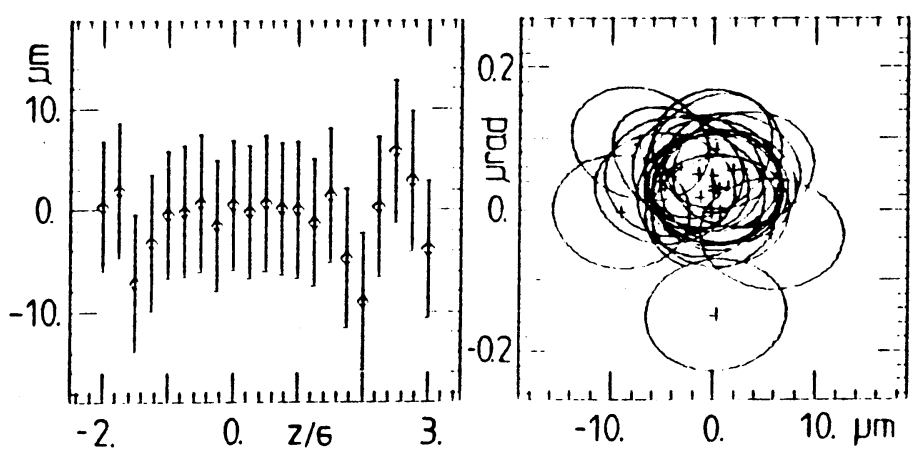

Figure 3: Slice positions and phase plot of a bunch at the end of the linac (AG focusing, corrected lateral quadrupole jitter of $1 \mu \mathrm{m}$ r.m.s. value)

damped after $200 \mathrm{~m}$ (Fig. 4b) and the emittance increase stops (Fig. 4c). The same strong damping occurs in the case of AG focusing.

\section{Conclusions}

Injection errors and positioning errors of RF structures are well approximated by smooth focusing. Lateral displaced quadrupoles however cause much larger betatron amplitudes in the case of AG focusing and therefore stronger wake field effects. Thus, in situations where BNS damping is required the smooth focusing may be one order of magnitude too optimistic.

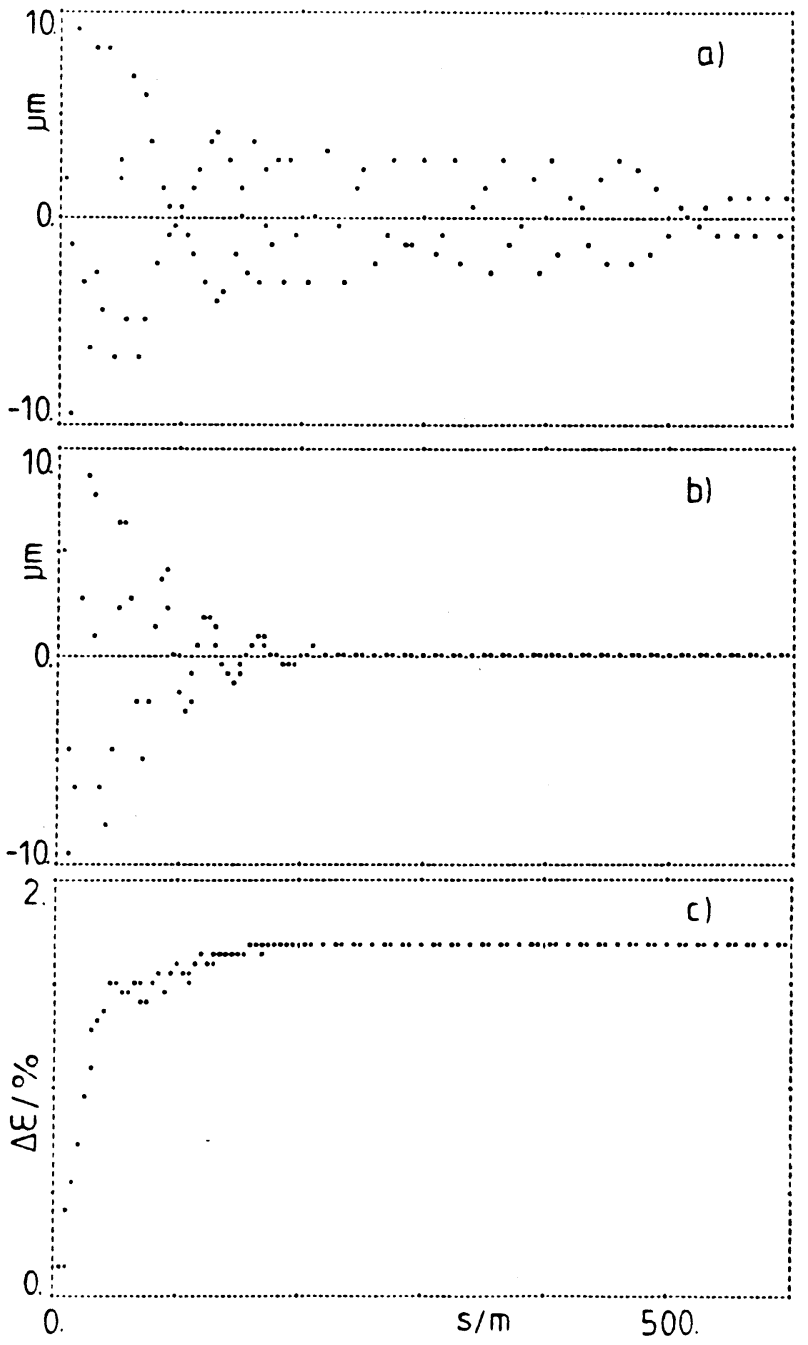

Figure 4: Centre of charge motion along the linac (smooth focusing, $10 \mu \mathrm{m}$ injection off-set). emittance growth for case b)

\section{References}

[1] V.E. Balakin, A.V. Novokhatsky and V.P. Smirnov, Proc. 12th Internat. Conf. on High Energy Accelerators, Batavia, 1983 (Fermilab, Batavia, 1984), p. 119.

[2] H. Henke, Proc. IEEE Particle Accelerator Conf., Washington 1987 (IEEE, New York, 1987), p. 1346.

[3] H. Henke, CERN-LEP-RF/88-45 (1988).

4) J. Tückmantel, CERN CLIC Note 87 (1989).

5) K.L.F. Bane, SLAC-PUB-3670 (1985).

[6] H. Henke and W. Schnell, CERN-LEP-RF/86-18 (1986).

(7) J.T. Seeman, Proc. 1988 Linear Accelerator Conf., Williamsburg 1988.

[8] W. Schnell, CERN CLIC Note 34 (1987).

[9] W. Schnell, CERN CLIC Note 56 (1987). 